\title{
Does the HD 209458 planetary system pose a challenge to the stellar atmosphere models?
}

\author{
A. Claret \\ Instituto de Astrofísica de Andalucía, CSIC, Apartado 3004, 18080 Granada, Spain
}

e-mail: claret@iaa.es

Received 4 May 2009 / Accepted 8 July 2009

\section{ABSTRACT}

\begin{abstract}
Aims. There are very few comparisons between theoretical and empirical limb-darkening coefficients (LDCs). To develop this scenario, we analyse the case of the HD 209458 planetary system for which 10 passband measurements of linear and quadratic LDCs are available.

Methods. The empirical data for HD 209458 were derived from the light curves obtained with the Hubble Space Telescope (HST). Since the corresponding effective wavelengths differ from those usually adopted, we computed monochromatic calculations to gain some insight into the problem. The theoretical LDCs were computed by adopting the least-squares method (LSM) but with a larger numerical resolution (100 $\mu$ points instead of 11). The flux conservation method (FCM) was also applied. The plane-parallel stellar atmosphere model ATLAS and the spherically symmetrical PHOENIX model were adopted in the calculations.

Results. We found systematic disagreement between the theoretical and empirical LDCs for the linear case. Disagreements are also found when we compared the quadratic LDCs with observations. Even taking into account uncertainties in the metallicity, microturbulent velocity, and effective temperature in the calculation of the theoretical LDCs, the corresponding shifted curves cannot match the empirical data. It seems that the current atmosphere models are unable to explain the specific intensity distribution of HD 209458.
\end{abstract}

Key words. stars: atmospheres - planetary systems

\section{Introduction}

Stellar physics still exhibits severe discrepancies when we compare its theoretical predictions with the available observational data. Some of these disagreements occur in studies of eclipsing binaries. For example, the system DI Her, whose apsidalmotion rate seems to present a problem for General Relativity (see for example, Claret 1998), or the case of low mass binaries $\left(0.7-1.1 M_{\odot}\right)$, whose observed radii are larger than those predicted by evolutionary models (Popper 1997; Clausen et al. 1999).

The comparison of eclipsing binary data with theoretical predictions involving atmosphere models has been limited because of the scarcity of information about limb and gravitydarkening. In a previous paper, we presented theoretical limbdarkening coefficients (LDCs) which have been calculated using standard least-squares method (LSM) for $100 \mu$ points (Claret 2008). These LDCs were compared with previous calculations of LDCs based on only $11 \mu$ points that we had previously published (Claret 2000) and with those derived by adopting the $r$-integration method. In terms of flux conservation, the average improvement is around one order of magnitude. With these specific calculations, we have compared theoretical with the few available empirical linear LDCs measured for nine eclipsing binaries. This comparison highlighted some discrepancies between the theoretical and observational data. These disagreements may be related to intrinsic problems in either the ATLAS models or the numerical methods adopted (including the chosen functions) to derive the theoretical LDCs. However, it may also indicate that the measurements are not accurate enough or exhibit systematic effects. At the true level of accuracy and given that the empirical LDCs are only available for 3 filters, it is impossible to identify more precisely the causes of this discrepancy.

As mentioned, the data from these nine eclipsing binaries are scarce and rather disperse. The observational data are only in $B V$ bands and for the $R$ band, only one empirical determination is available. This limits the quality of our conclusions significantly, although a dependence on the effective temperature was detected. On the other hand, Southworth $(2008,2009)$ presented a comprehensive and homogeneous determination of light curves and physical properties of fourteen transiting extrasolar planetary systems. For one parent star (HD 209458), the linear and quadratic LDCs were directly determined from the HST light curves. The advantage of this determination is that the number of wavelengths used (10) is higher than those often obtained for eclipsing binaries. The covered range in $\lambda$ is 320 up to $980 \mathrm{~nm}$. An extra and important advantage is that the reflection effect which in ordinary eclipsing binaries can complicate the derivation of the LDCs, is negligible in these cases. In this paper, we compare more deeply the empirical LDCs for HD 209458 with theoretical calculations by adopting different numerical methods and atmosphere models.

\section{Numerical methods and atmosphere models}

In the present paper, we compute the theoretical LDCs by adopting two numerical methods: the standard LSM with $100 \mu$ points, and the flux conservation method (FCM). The laws which most frequently used in limb-darkening studies are: the linear (coefficient $u(\lambda)$ ), the quadratic (coefficients $a(\lambda)$ and $b(\lambda)$ ), the square root, the logarithmic, and a law with five terms introduced by Claret (2000). These laws are well known and not reproduced 
here. The LDCs are often computed for some determined passbands but here we compute the monochromatic values to obtain a clearer insight into the problem. We adopt the plane-parallel atmosphere models ATLAS (Kurucz 2000) and the spherically symmetrical LDCs presented in Claret \& Hauschildt (2003).

\section{The HD 209458 planetary system: comparison with theoretical predictions of LDCs}

HD 209458 was the first transiting extrasolar planet discovered (Charbonneau et al. 2000). The mass of the parent star is $1.165 \pm$ $0.033 M_{\odot}$, its effective temperature is $6117 \pm 50 \mathrm{~K}$, its $\log g=$ $4.368 \pm 0.005$, and its metallicity is slightly higher than the solar value $([\mathrm{Fe} / \mathrm{H}]=0.02 \pm 0.05)$ (Santos et al. 2004; Southworth 2009). Since we consider the error bar in the effective temperature to be too low, we assume here an error of $250 \mathrm{~K}$, which seems to be more realistic. The derived age is $2.3_{-0.6}^{+0.7} \mathrm{Gyr}$ by using stellar evolutionary models (Southworth 2009).

\subsection{The linear approach}

Basically, there are four factors that could influence the comparison between the theoretical and empirical LDCs. The first is the degeneracy in the fitting of the transit light curves, which may cause systematic errors in the LCDs. The information contained in transit light curves is not optimal, and the LDCs of twoparameter laws are usually correlated (see for example Fig. 2 in Southworth 2008). However, this does not necessarily cause a systematic error. In the case of the linear law, the corresponding LDCs are not affected by this degeneracy. We can consider that empirical LDCs obtained by Southworth (2008) are free from the degeneracy in the fitting of the transit light curves. The second factor is related to the kind of function used to fit the stellar limb-darkening. As mentioned, Southworth (2008) obtained empirical linear and quadratic LDCs. We focussed our attention on these two laws. The linear law, in general, can be ruled out by very high quality observations (recall also that the limb-darkening is a non-linear phenomenon). In addition, for the linear case, Southworth (2008) found that the orbital inclination is correlated with the wavelength of observation, which clearly highlights the inadequacy of the linear law. For the quadratic case, no correlation was found. The bi-parametric laws (quadratic, cubic, root-square, and logarithmic) produce almost identical fit qualities (Southworth, personal communication). For completeness, we investigate the linear and the quadratic cases here by adopting the ATLAS and PHOENIX models and considering two numerical methods: the LSM and the FCM. The third factor that could influence the comparison between the theoretical and empirical LDCs is the size of the error bars in the effective temperature, $\log g$, and metallicity may also affect the comparison between theoretical and empirical LDCs. We carried out a detailed comparison between theory and observations by considering the mentioned error bars. Finally, and perhaps the most important factor, we should that consider are the limitations of the stellar atmosphere models in predicting the specific intensity distribution.

Southworth (2008) compared the empirical linear LDCs for HD 209458 (the corresponding error bars come from the Monte Carlo simulations) with those derived from the atmosphere models. As a theoretical support, he adopted the LDCs computed for the ATLAS model (SLOAN photometric system). However, a more complete comparison with theoretical predictions was not carried out because this comparison was beyond
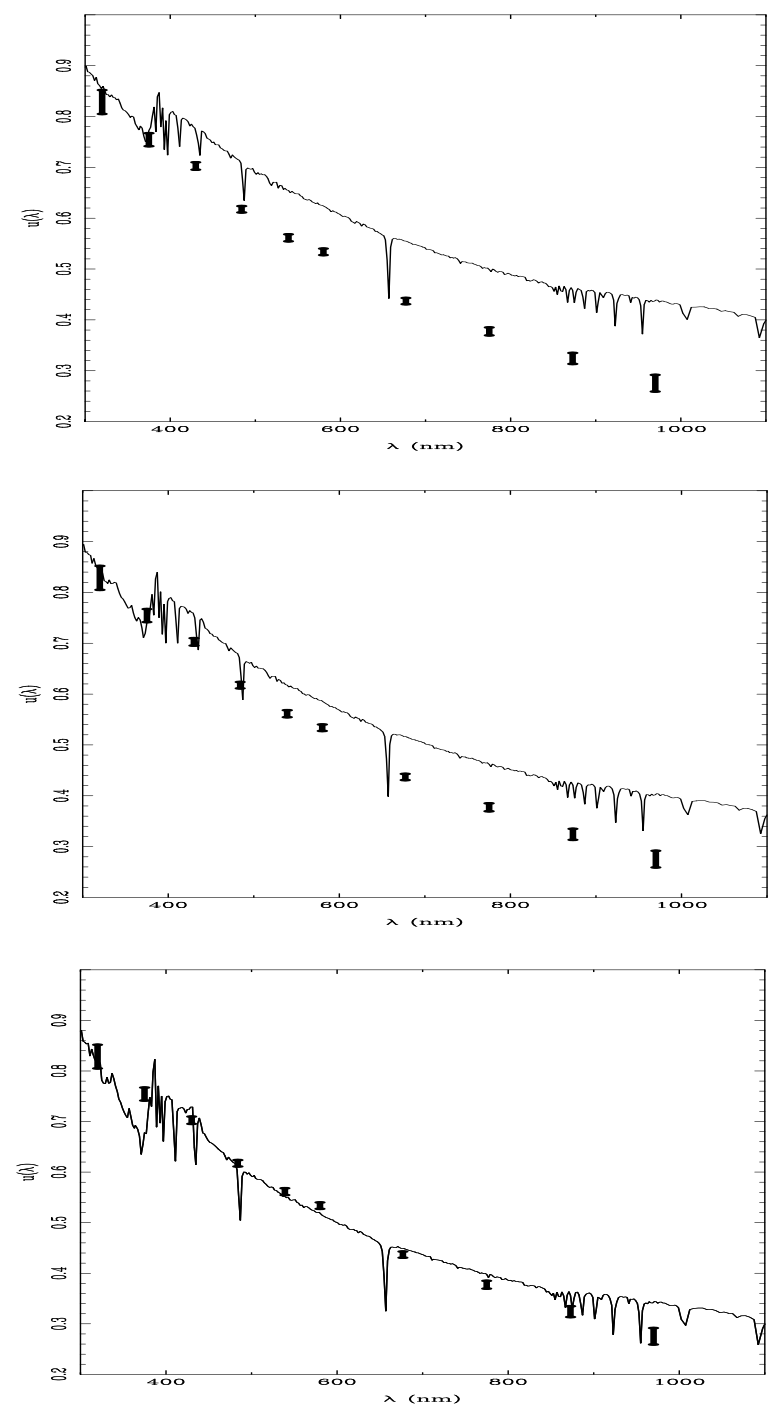

Fig. 1. Comparison between empirical linear LDCs for HD 209458 (error bars) and theoretical predictions (continuous line): a) theoretical values from Claret (2000) using $11 \mu$ points; b) theoretical values computed using $100 \mu$ points; and c) theoretical values computed by adopting FCM. All theoretical LDCs were computed by using the ATLAS models, $[\mathrm{m} / \mathrm{H}]=0.00, V_{\xi}=2 \mathrm{~km} \mathrm{~s}^{-1}$.

the scope of that paper. Here we compare the LDCs by using more theoretical tools. In the first panel of Fig. 1 we show the same comparison as that by Southworth for the linear law. In that figure, we adopted monochromatic calculations instead of taking into account the filters transmission to gain some deeper insight given that the shortest effective wavelength of the photometric bands usually adopted is longer than $320 \mathrm{~nm}$ (the lower limit for the HST passbands). We note that there are differences between the theoretical and empirical LDCs that are consistent with Southworth's comparison; the theoretical LDCs are systematically larger than the empirical ones. For shorter wavelengths, the differences are small and we can even accept that, within the errors, the theoretical values fit the observations. However, for wavelengths longer than $400 \mathrm{~nm}$, the differences increase. Is this indicative of a problem with the atmosphere models at longer wavelengths? Before trying to answer this question we explore the numerical methods used to derive the theoretical LDCs. The second panel of Fig. 1 shows the same comparison but for when 

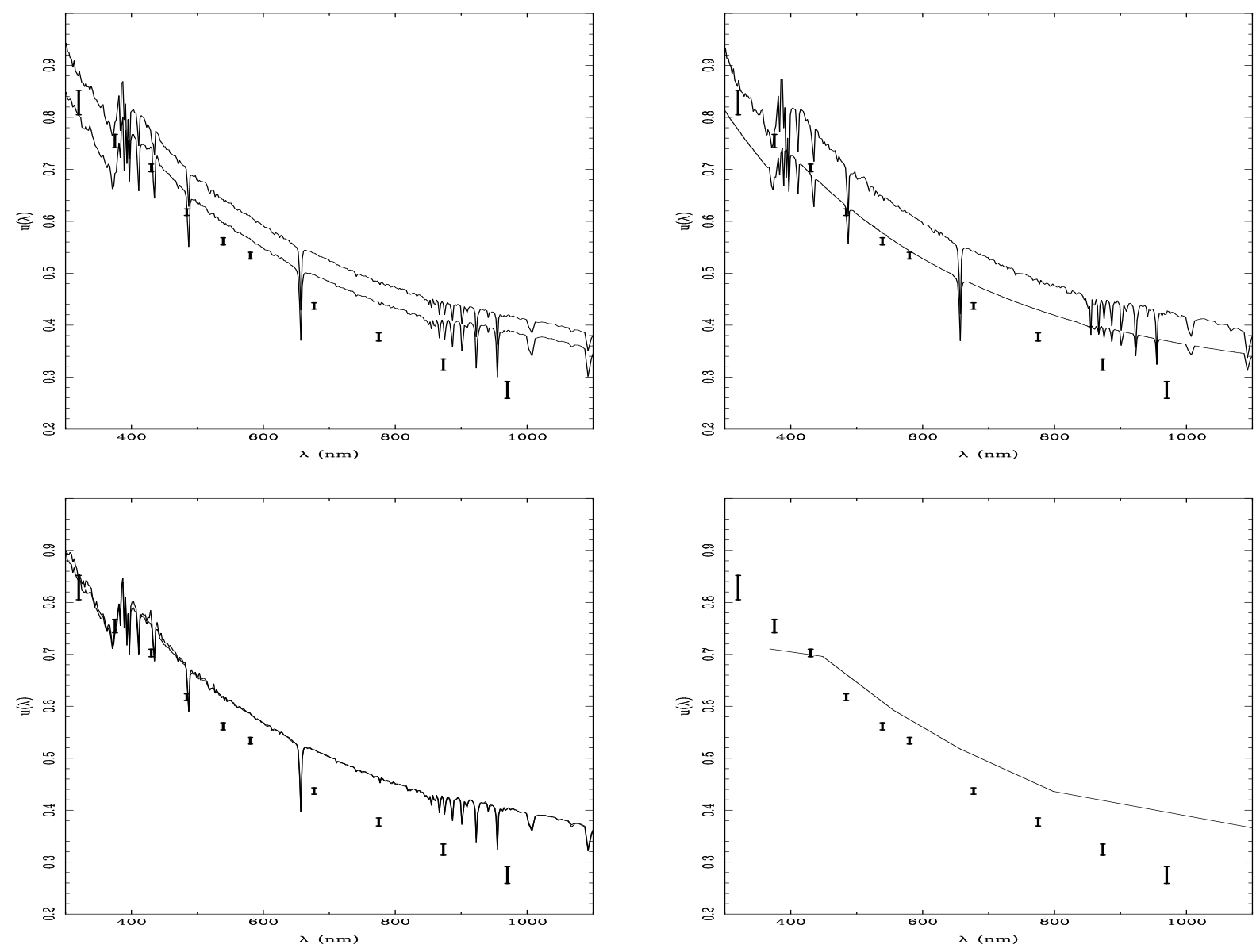

Fig. 2. Comparison between empirical linear LDCs for HD 209458 (error bars) and theoretical predictions (continuous line). a) Theoretical values computed using $100 \mu$ points and with $\Delta T_{\text {eff }}= \pm 250 \mathrm{~K}$; b) theoretical values computed using $100 \mu$ points and with [m/H] $= \pm 0.50$; c) theoretical values computed using $100 \mu$ points and $V_{\xi}=2$ and $8 \mathrm{~km} \mathrm{~s}^{-1}$. ATLAS models; and d) spherical models (PHOENIX), $V_{\xi}=2 \mathrm{~km} \mathrm{~s}{ }^{-1}$ and $[\mathrm{m} / \mathrm{H}]=0.00$.

the number of $\mu$ points are increased to 100 (instead of 11). The same systematic effect is still detected, although on a small scale.

Generally, for a given atmosphere model, the FCM produces smaller linear LDCs than those computed by using the LSM (Claret 2008). The third panel of Fig. 1 compares the LDCs for HD 209458 evaluated by adopting the FCM. The disagreements clearly decrease at all wavelengths. However, as has been shown in several papers, the FCM does not represent well the specific intensities and produces very high values of $\sigma$ s. By definition, this method preserves integrally the flux. This should be an advantage but in contrast, if we analyse the situation in more detail we see that it is a disadvantage. The limb-darkening is clearly a non-linear phenomenon. So, the FCM is unable to find the best function because it always preserves the flux. In contrast, the LSM produces large $\sigma$ s and $\delta$ Fs (the ratio of the true flux at the wavelength $\lambda$ and that using a given limb-darkening law) when we adopt the linear approximation and provides a robust means of differentiating between the functions chosen to fit the stellar limb-darkening. For a more detailed discussion of this subject, we refer the readers to Claret (2000).

The observational error bars in the effective temperature of the parent star need to be considered when comparing with theoretical predictions. In the first panel of Fig. 2 we show the theoretical LDCs evaluated by taking into account error bars of $250 \mathrm{~K}$. Even considering these error bars, the shifted curves of the theoretical LDCs are incapable of matching the observations. On the other hand, the chemical composition is not well determined in most of the stellar systems. We computed LDCs by varying the metal content by $0.50 \mathrm{dex}$ and the results are shown in the second panel of Fig. 2. Although the theoretical LDCs with $[\mathrm{m} / \mathrm{H}]=-0.50$ somewhat improve the comparison mainly at shorter wavelengths, they are unable to fit the observational data satisfactorily.

Another parameter that can be explored is the microturbulent velocity $V_{\xi}$ but even by increasing this to $8 \mathrm{~km} \mathrm{~s}^{-1}$, the empirical LDCs remain smaller than the resulting theoretical ones (third panel of Fig. 2). Finally, in the fourth panel of Fig. 2, we show the calculations considering the spherical PHOENIX models (Claret \& Hauschildt 2003). There is no large difference from the panels 1 and 2 of Fig. 1, but the discrepancies are slightly smaller. This may indicate that PHOENIX models can represent the atmosphere of HD 209458 better than the ATLAS models. We note that for this model we adopted the $U B V R I J H K$ passbands instead of monochromatic calculations.

\subsection{The quadratic approach}

Southworth (2008) also obtained LDCs for the quadratic law case. As in the previous case, the Monte Carlo simulations were carried out to estimate the error bars. Figure 3 shows the comparison between the empirical quadratic LDCs and the theoretical calculations for the ATLAS model, with $[\mathrm{m} / \mathrm{H}]=0.00$, $V_{\xi}=2 \mathrm{~km} \mathrm{~s}^{-1}$. The comparison highlights some differences with 

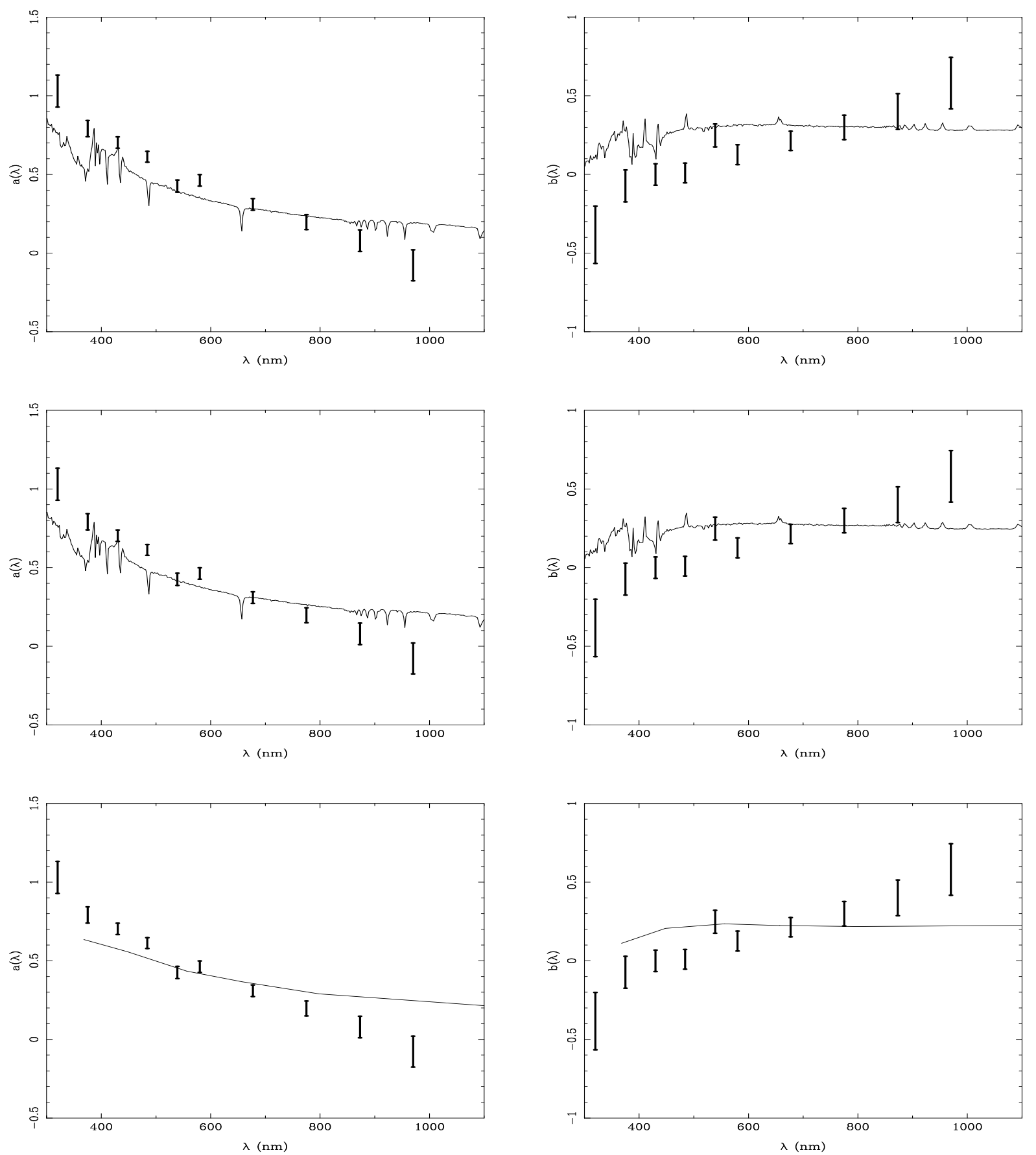

Fig. 3. Comparison between empirical quadratic LDCs for HD 209458 (error bars) and theoretical predictions (continuous line): a) theoretical values computed using $100 \mu$ points, linear component; b) theoretical values computed using $100 \mu$ points, quadratic component; c) theoretical values computed using FCM, linear component; d) theoretical values computed FCM; quadratic component, ATLAS models, $V_{\xi}=2 \mathrm{~km} \mathrm{~s}^{-1}$, $[\mathrm{m} / \mathrm{H}]=0.00 ; \mathbf{e})$ spherical models (PHOENIX), linear component; f) spherical models (PHOENIX), quadratic component, $V_{\xi}=2 \mathrm{~km} \mathrm{~s}^{-1}$ and $[\mathrm{m} / \mathrm{H}]=0.00$.

respect to the linear case. In the last panel, we found a systematic disagreement with wavelength. For the quadratic approximation, the theoretical linear components $a(\lambda)$ are larger than the empirical ones for wavelengths longer than $800 \mathrm{~nm}$ and smaller than the observed for $\lambda$ shorter than $600 \mathrm{~nm}$ (first panel). Only for the interval 700-800 $\mathrm{nm}$ do the theoretical linear components seem to fit the observations. A more conspicuous difference is found when we compare the theoretical and empirical quadratic component $b(\lambda)$ (second panel of Fig. 3). The theoretical $b(\lambda)$ is almost independent of wavelength, while the empirical values exhibits a pronounced dependence, being negative at shorter wavelengths. As pointed out by Southworth (2008), this characteristic is not present in any theoretical predictions that he adopted. To check whether this also holds for other numerical methods, we also compare the $a(\lambda)$ and $b(\lambda)$ obtained by using the FCM with the observed ones (panels 3 and 4, Fig. 3). The theoretical LDCs generated by using the FCM are unable to fit the observed $a(\lambda)$ and $b(\lambda)$. Finally, we compare the observations with the predictions from the spherical models (panels 5 and 6, Fig. 3). The aspects of panels $1-6$ of Fig. 3 are similar, 

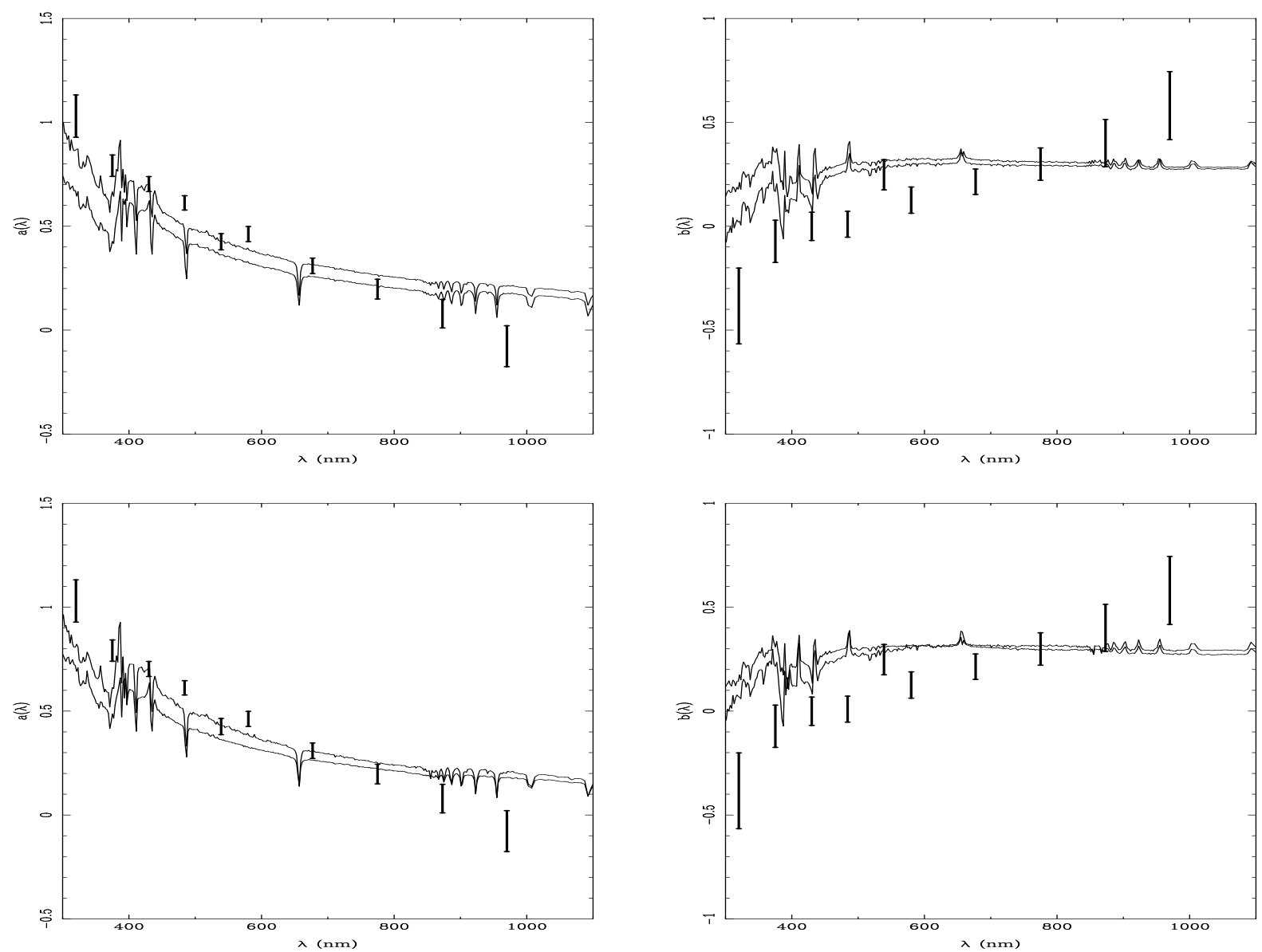

Fig. 4. Effects of uncertainties in both $T_{\text {eff }}( \pm 250 \mathrm{~K})$ and $[\mathrm{m} / \mathrm{H}]( \pm 0.50)$ on the quadratic LDCs for HD 209458 (error bars) and theoretical predictions (continuous line): a) theoretical values computed using $100 \mu$ points, linear component, $\Delta T_{\text {eff }}= \pm 250 \mathrm{~K}$; b) theoretical values computed using $100 \mu$ points, quadratic component, $\Delta T_{\text {eff }}= \pm 250 \mathrm{~K} ; \mathbf{c}$ ) theoretical values computed using $100 \mu$ points, linear component, $\Delta[\mathrm{m} / \mathrm{H}]= \pm 0.50$; d) theoretical values computed using $100 \mu$ points, quadratic component, $\Delta[\mathrm{m} / \mathrm{H}]= \pm 0.50$, ATLAS models.
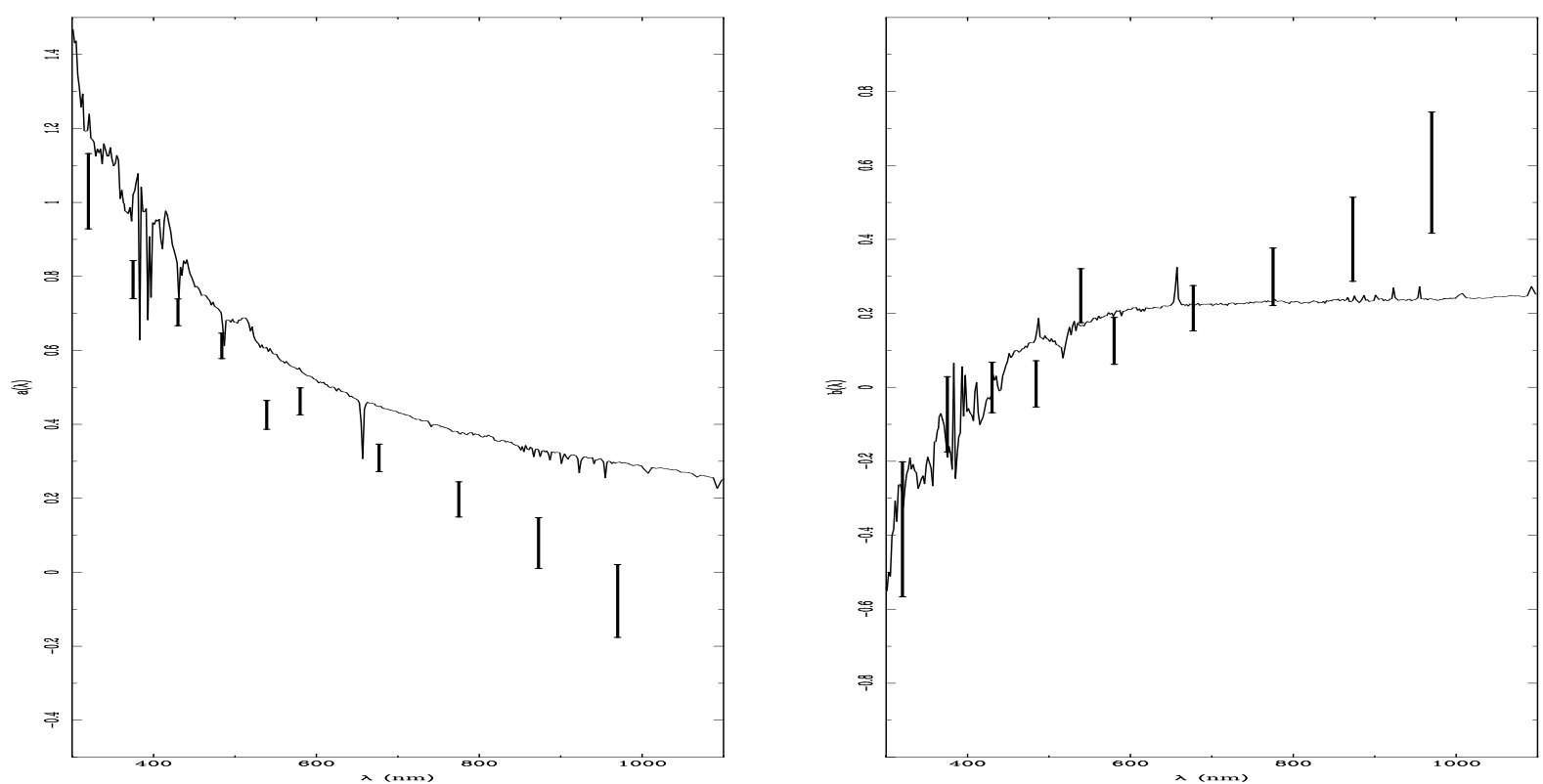

Fig. 5. Comparison between empirical quadratic LDCs for HD 209458 (error bars) and theoretical predictions (continuous line): a) theoretical values computed using $100 \mu$ points, linear component; b) theoretical values computed using $100 \mu$ points, quadratic component, $T_{\text {eff }}=5250 \mathrm{~K}$, $\log g=4.5$ and $V_{\xi}=2 \mathrm{~km} \mathrm{~s}^{-1}$, ATLAS model.

and it seems that only if we consider a "rotation" of the theoretical linear and quadratic components around $\lambda=550 \mathrm{~nm}$ would it be possible to reproduce the observed quadratic LDCs. Even if we take into account the effects of uncertainties in $T_{\text {eff }}$ and 
in $[\mathrm{m} / \mathrm{H}]$, the general aspect of Fig. 3 does not change much. Figure 4 shows these comparisons. We note that while the theoretical linear components that take account of the uncertainties in both $T_{\text {eff }}$ and [m/H] (panels 1 and 3 ) improve the comparison, the quadratic components are not affected significantly by the error bars.

Since the previous comparisons indicate that the atmosphere model with the observed $T_{\text {eff }}$ and $\log g$ is unable to reproduce the empirical LDCs of HD 209458, we decided to search for a model that could match the measurements more successfully to compare its physical properties with the observed $T_{\text {eff }}$ and $\log g$. The ATLAS model that matches the quadratic LDCs more closely is that with $T_{\text {eff }}=5250 \mathrm{~K}, \log g=4.5,[\mathrm{~m} / \mathrm{H}]=0.00$, and $V_{\xi}=2 \mathrm{~km} \mathrm{~s}^{-1}$ and is represented in Fig. 5 with the data for HD 209458. The agreement can be considered to be acceptable only for $\lambda \mathrm{s}$ shorter than $450-500 \mathrm{~nm}$, but for longer wavelengths the disagreement is clear and similar to that displayed in the first and second panels of Fig. 1 for the linear component. The theoretical quadratic components marginally fit the observations for $\lambda$ s shorter than $800 \mathrm{~nm}$ but fail to match them at longer wavelengths. In addition, the effective temperature of this exploratory model is too low compared with the observational one, even taking into account the error bars adopted here $( \pm 250 \mathrm{~K})$.

\section{Final remarks}

From the above comparisons, we can conclude that the current stellar atmosphere models are unable to predict the measured LDCs for HD 209458. The unique case that seems to fit the observations marginally is the linear approach (Sect. 3.1) when we adopt the FCM. However, the limb-darkening is clearly a nonlinear phenomenon and this law is then inadequate. For FCM, this method, which preserves the flux integrally, is inadequate because it does not describe well the specific intensities. As is already known, for a given atmosphere model, the FCM provides smaller linear LDCs than those computed by adopting the LSM. This is probably the reason why the linear theoretical LDCs computed by using the FCM seem to reproduced more successfully the empirical values (third panel, Fig. 1). When we apply the FCM to the quadratic law, the corresponding LDCs also do not match the observations (panels 3 and 4, Fig. 3). In this way, the exact flux conservation condition seems to be disconnected from the goodness of the fitting.

The disagreements are also present when we adopt the quadratic law. We have explored the effects of the error bars in the effective temperature, metallicity, and micro-turbulent velocity in the prediction of the theoretical LDCs. The shifted curves are not enough to explain the measured values.

In addition to the different numerical methods, we have tested two sets of stellar atmosphere models: ATLAS and PHOENIX. Both models, for the direct comparison with the empirical LDCs, provide similar theoretical predictions and none is able to match the observations. This indicates that the current atmosphere models need updating and that probably, given the effective temperature of HD 209458, these modifications should involved the process of convective transport of energy and related to the opacities tables.

The impact of these disagreements on the derived masses and on the radii of transiting extrasolar planets can be large, achieving 3 to $5 \sigma$ in the case of the ratio of the radii. New highaccuracy light curves are necessary for the transiting extrasolar planetary systems. The two alternatives, including the LDC as fitted parameters and taking them directly from the stellar atmosphere models, should be compared. On the other hand, other fields of the stellar astrophysics may also be affected by the inability of the stellar atmosphere models to reproduce correctly the intensity distribution, at least for the range of effective temperature studied here. It would desirable that the impact of the disagreement to be investigated and confirmed (or not) in other independent fields of astrophysics.

Acknowledgements. I would like to thank J. Southworth, J. V. Clausen, M. A. Valverde, V. Costa, G. Torres and an anonymous referee for useful discussions. The Spanish MEC (AYA2006-06375) is gratefully acknowledged for its support during this work.,

\section{References}

Charbonneau, D., Brown, T. M., Latham, D. W., \& Mayor, M. 2000, ApJ, 529, L45

Claret, A. 1998, A\&A, 330, 533

Claret, A. 2000, A\&A, 363, 1081

Claret, A. 2008, A\&A, 482, 259

Claret, A., \& Hauschildt, P. H. 2003, A\&A, 412, 241

Clausen, J. V., Baraffe, I., Claret, A., \& VandenBerg, D. A. 1999, ASP Conf. Ser. 173, ed. A. Giménez, E. F. Guinan, \& B. Montesinos, 265

Kurucz, R. L. 2000, private communication

Popper, D. M. 1997, AJ, 114, 1195

Santos, N. C., Israelian, G., \& Mayor, M. 2004, A\&A, 415, 1153

Southworth, J. 2008, MNRAS, 386, 1644

Southworth, J. 2009, MNRAS, 394, 272 